Quintriqueo, S., Quilaqueo, D., Lepe-Carrión, P., Riquelme, E., Gutiérrez, M. \& Peña-Cortes, F. (2014). Formación del profesorado en educación intercultural en América Latina. El caso de Chile. Revista Electrónica Interuniversitaria de Formación del Profesorado, 17 (2), 201-217l.

DOI: http://dx.doi.org/10.6018/reifop.17.2.198021

\title{
Formación del profesorado en educación intercultural en América Latina. El caso de Chile
}

Segundo Quintriqueo, Daniel Quilaqueo, Patricio Lepe-Carrión, Enrique Riquelme, Maritza Gutiérrez, Fernando Peña-Cortés

Universidad Católica de Temuco

\section{Resumen}

El artículo tiene como objetivo analizar la educación intercultural y la formación del profesorado en América Latina, especialmente en Chile. El tema es abordado desde una reflexión del Equipo de Investigadores titulares del Centro de Investigación en Educación en Contexto Indígena e Intercultural (CIECII), de la Iniciativa Científica Milenio (ICM), de la Universidad Católica de Temuco. Sostenemos que la interculturalidad en América Latina tiene su origen en la relación dinámica y compleja entre las propias comunidades indígenas y en las posteriores transformaciones socioculturales legadas en el proceso de conquista, colonización y constitución de los Estados-Nación. En este contexto, la educación intercultural bilingüe ha sido históricamente pensada para los indígenas, en tanto que la escolarización ha sido concebida principalmente como estrategia instrumental de los Estados-Nación, para continuar con los procesos de dominación heredados desde la colonia (colonialidad), especialmente en la institucionalidad educativa. Así, pensar la interculturalidad de manera crítica, es concebirla como un proyecto ético, político y epistémico a construir, para la transformación de los fundamentos que sostienen la asimetría y desigualdad en nuestra sociedad y que se reproduce a través de la escolarización. Concluimos que para la formación del profesorado, la interculturalidad debería tener como finalidad construir el diálogo entre sujetos que pertenecen a sociedades y culturas diferentes, para contrarrestar la linealidad de la monoculturalidad, superar el carácter colonial de la acción educativa, en base a prácticas pedagógicas fundadas en la conciencia crítica, así como la lucha contra el racismo y la discriminación. 


\title{
Palabras clave
}

educación intercultural; formación del profesorado; diversidad cultural; epistemología.

\section{Teaching training in intercultural education in Latin America. The Chilean case}

\begin{abstract}
This article aims to analyze the intercultural education and teaching formation in Latin America, specially in Chile. The study of this subject is an analysis of the Team of Senior Researchers of the Research Center in Indigenous and Intercultural Context (CIECII)- "Centro de Investigación en Educación en Contexto Indígena e Intercultural" - part of the Millennium Scientific Initiative (ICM in Spanish) program by the Universidad Católica de Temuco. We uphold the view that interculturality in Latin America had its origin in the dynamic and complex relationships among the different indigenous communities and in the subsequent sociocultural transformations that resulted from the processes of conquest, colonization and constitution of the nation-states. In this context, bilingual intercultural education has been historically aimed at indigenous people, while formal schooling has been conceived mainly as a strategic tool of the nation-states to continue the dominance processes inherited from the colonial time (coloniality), specially in the school institutions. Thus, thinking about interculturality in a critical manner means to conceive it as an ethical, political and epistemological project to be. A project that would transform the foundations that underpin the asymmetries and inequities in our society and that are replicated through formal schooling. We can conclude that, for teaching training, interculturality should seek to build a dialogue among subjects belonging to different societies and cultures in order to counteract the single-lined monoculturalism, to overcome the colonial characteristics of the educational actions, based on teaching practices based on critical awareness, as well as to combat racism and discrimination.
\end{abstract}

\section{Key words}

Intercultural education; teaching training; cultural diversity; epistemology.

\section{Introducción}

Para comprender y explicar la formación del profesorado en educación intercultural, en el presente artículo, sostenemos que los pueblos indígenas en América Latina han construido sus saberes sobre la base de una educación propia-familiar, en una relación dinámica y compleja entre sus comunidades. Estas son características socioculturales que, en el proceso de conquista, colonización y constitución de los Estados-Nación, para el caso de Chile, han sido históricamente negadas y/o subalternizadas. Por ejemplo, la usurpación de tierras, la esclavitud y procesos de minorización cambiaron sus modos de vivir. Producto de la imposición cultural por medio de la evangelización y la educación escolar, cambiaron 
también su modo de conocer. Así, en base a una educación orientada a la utilidad del indígena, se logró su integración a la sociedad chilena como sujetos de trabajo. Es por ello que, tanto en Chile como en América Latina, no es suficiente una mirada desde el problema de la migración o tipos de diversidad cultural para debatir sobre interculturalidad. Más bien, se requiere prestar atención a la experiencia de dominación en la que han estado inmersos los pueblos indígenas, hasta hoy día. Es decir ¿cómo estos pueblos fueron inventados y producidos como inferiores respecto a la población de origen europeo que colonizara América Latina? En esta perspectiva, es posible pensar la emergencia, desarrollo y orientación de la educación intercultural en América Latina, especialmente en Chile, y sobre todo del rol transformador y crítico que debe tener la formación del profesorado en contexto indígena en la actualidad.

El artículo comienza con una breve historización del problema de la subalternidad en Chile, es decir, cómo se presenta desde la sociedad chilena no indígena el discurso de inferiorización sobre los pueblos indígenas, específicamente en el caso mapuche. Esto permite contextualizar la idea de interculturalidad como una propuesta crítica y no funcional al sistema educacional actual, que no sólo legitima la diferencia y desigualdad, sino que además, la reproduce como tal (Baudelot y Leclerq, 2008). Luego, en una segunda parte, se presenta la formación de profesores en educación intercultural en América Latina, para focalizarse en la formación del profesorado en educación intercultural en Chile. En este proceso se destacan aquellos factores que han marcado hitos en esta formación.

\section{Pensar desde una interculturalidad crítica}

Sería muy extenso hacer un repaso detallado de los distintos procesos históricos por los cuales se instaló en Chile una matriz colonial de poder sustentada en la idea de raza como eje central en la articulación de las relaciones interétnicas (Quilaqueo y Merino, 2003; Merino, Mellor, Quilaqueo y Saiz, 2008; Quintriqueo, 2010; Lepe-Carrión, 2012a, 2012b). El concepto de raza que empleamos aquí es restringido, puesto que nada tiene que ver con la pseudocientífica definición de finales del siglo XIX. Se entiende más bien, como una interpretación y construcción social que tiene relación con un imaginario cultural que clasifica y jerarquiza a la sociedad sobre la base de una anulación e imposición de nuevos códigos de existencia (Quilaqueo, Merino y Saiz, 2007; Lepe-Carrión, 2012a). En esa perspectiva, la cultura europea será ubicada como el prototipo de civilización, mientras que las culturas de los pueblos indígenas constituirán un modo de vivir retardado, 'bárbaro' e incluso inhumano. Este modo de clasificación social, constituirá un antecedente a las posteriores formas de jerarquización (De la Cadena, 2007; Burns, 2007; Arias Y Restrepo, 2010).

A partir de la clasificación señalada, es posible determinar como hito fundador del proceso de producción de la inferiorización de los indígenas, en el reino de España en Chile, la guerra defensiva que acontece a los pocos años del triunfo en la Batalla de Curalaba en 1598 (LepeCarrión, 2012a). Sin embargo, los españoles habían perdido gran parte de las ciudades y soldados debido al masivo levantamiento de los indígenas (Bengoa, 1987). Así, la muerte del gobernador García Oñez de Loyola fue quizás la más simbólica de las pérdidas de los conquistadores españoles en aquel entonces. En este contexto, en la colonia del reino de España en Chile, hubo un revuelo entre la élite, condenándose enérgicamente la sublevación de los indígenas mapuches por una parte y, los intentos por reactivar la legalización de la esclavitud indígena, por otra parte. Esta última idea se venía practicando desde hace años, pero de manera ilegal. Con la creación de la Tasa de Santillan y luego de Gamboa, se reemplazó la esclavitud por una pragmática forma de explotación legal en el trabajo (Jara, 1971; Hanisch, 1974). Sin embargo, este debate fue infructuoso y ocurrió algo 
muy similar a lo acontecido años antes en la disputa de Valladolid (Célebre debate que tuvo lugar entre 1550 y 1551 en el Colegio de San Gregorio de Valladolid, dentro de la llamada polémica de los naturales en referencia a los indígenas americanos y que enfrentó dos formas antagónicas de concebir la conquista de América Latina. Por una parte, los defensores de los indios representado por Bartolomé de las Casas y por otra parte, los enemigos de los indios, representado por Juan Ginés de Sepúlveda, que defendía el derecho a la conveniencia del dominio de los españoles sobre los indígenas y los concibe naturalmente inferiores). Pero en este caso, el sacerdote jesuita Luis de Valdivia encabezaba las demandas por disminuir la violencia y aprovechar la existencia de los 'bárbaros' como sujetos que puedan pasar de ser enemigos a súbditos del rey (Bengoa, 1987). Luis de Valdivia, se percató que la guerra ofensiva no sólo era costosa para el arca del reino español tanto humana como económicamente, sino que además lo era en términos de efectividad en el tiempo, pues los indígenas se volvían cada vez más violentos. Fue así como propuso al rey un proyecto de guerra defensiva donde la evangelización del mapuche permitiera su domesticación e inserción a la lógica de la civilización occidental (Bengoa, 1987). Es decir que la diferencia colonial-racial fuera reemplazada, o más bien encubierta, por una diferencia cultural (Lepe-Carrión, 2012C).

El proyecto de guerra defensiva no tuvo los efectos esperados por los conquistadores. Sin embargo, se constata que este evento tuvo la capacidad de instalar en Chile una serie de dispositivos de dominación que vinieron a reemplazar los viejos mecanismos soberanos de quitar la vida, por otros que tenían que ver con el disciplinamiento o subjetivación del indígena como un sujeto colonial (Cano, 2010; Lepe-Carrión, 2012a, 2012b). Las misiones, los parlamentos, las escuelas de indios, las técnicas de comercio, la designación de caciques embajadores, entre otras, eran todas estrategias de vigilancia, registro y control. Estas estrategias permitían al colonizador no solamente estar en conocimiento de lo que sucedía al interior del territorio que estaba más allá de la frontera, sino que además, operar en las mentalidades de los indígenas. Es decir, que la evangelización o escolarización servía como un eficaz instrumento de dominación, por el cual se vehiculará la diferencia racial en la producción de sujetos inferiorizados hasta bien entrado el siglo XIX con la formalización de la institucionalidad chilena (Boccara, 1996, 1999; Poblete, 2009; Lepe-Carrión, 2012a, 2012d).

En este contexto, proponemos pensar la interculturalidad como un proyecto educativo a construir, que nos permita desestructurar las herencias coloniales que subyacen en la estructura, en la institucionalidad y relaciones sociales (Quintriqueo y Mcginity, 2009; Quintriqueo, 2010; Quilaqueo, Fernández Y Quintriqueo, 2010; Ferrão, 2010). Este proceso tiene necesariamente que comenzar identificando las causas del no-diálogo, y no reducirse a un problema que comprometa solamente asuntos educacionales, sino que, también aspectos más trascendentales que involucren agencias mucho más amplias del Estado, como por ejemplo: la economía, la justicia, y por supuesto, la educación (Tubino, 2005a). Así, nuestra postura es que la interculturalidad pensada exclusivamente desde la constatación de la diversidad cultural o como el multiculturalismo (enfoque anglosajón), no es más que un modo funcional de la misma hacia la matriz colonial de poder, la hace legítima, y lo que es peor, la reproduce permanentemente en su discurso de inclusión o reconocimiento. En cambio, pensar la interculturalidad de manera crítica, es concebirla como un proyecto ético, político y epistémico para la transformación de los fundamentos que sostienen la asimetría y desigualdad en que se encuentra nuestra sociedad (Freire, 2002; Ferrão, 2010; Quintriqueo, Quilaqueo Y Torres, 2014). Especialmente en la educación en contexto de comunidades indígenas, significa ir más allá de los límites de la forma Estado liberal, moderno y mercantilizado, para que se tienda no solamente a la homogeneización y occidentalización. 
Desde nuestra postura, tampoco se trata de un proyecto para indígenas. Es en la occidentalización donde permanece esta idea de que son los 'indios' quienes deben entrar a la modernidad, como si en América Latina haya habido solo una recepción y tardía, 'de los goteos rebalsados' de modernización europea ya constituida (Quijano, 1988; De Sousa, 2010). Así, se desconocen los lugares propios desde los cuales ha sido experimentada la modernidad. En este sentido, la modernidad ha tenido discursos múltiples de producción y enunciación, y la conquista del nuevo mundo fue siempre el aliciente fundamental del imaginario europeizado de dominación: América se vuelve un lugar de futuras realizaciones espirituales y materiales, la edad dorada civilizatoria estaba por construir (Quijano, 1988; De Sousa, 2010). En ese contexto, los modelos de intervención estatal sobre 'lo indígena', conllevan esta visión de receptividad y los proyectos interculturales siguen pensando al indígena como sujeto central de la interculturalización, dejando al margen a quienes ya han asimilado la occidentalización (Tubino, 2005b; Walsh, 2010). Un proyecto de interculturalidad para todos, indígenas y no indígena, se constituye en un proyecto de sociedad, que no nace desde la diversidad en tanto constatación, sino más bien, su punto de partida se ubica en la diferencia colonial y cultural que ha sido heredada desde la construcción colonial de los Estados-Nación en América Latina (Ferrão, 2010). Es así como en Chile, la colonialidad aún permanece arraigada en las prácticas culturales y en la institucionalidad. Es allí donde se debería concebir un proyecto que nace 'desde abajo' o desde la subalternidad, pero no en el sentido de reivindicación de algún tipo de populismo político, ni menos en la nostalgia de volcarnos a una identidad estática del pasado, en una cultura inmutable. Nos referimos más bien a reinventar alternativas concretas al modelo monocultural imperante, principalmente en educación, por uno donde se promuevan otras formas de articular las diferencias, otras epistemologías que posibiliten nuevas formas de con-vivir, de pensar y de sentir (Tubino, 2005b, 2005a; Viaña, 2010; Walsh, 2010; Quintriqueo, Quilaqueo Y Torres, 2014).

\section{Sobre la emergencia institucionalizada de la educación intercultural}

Si la modernidad es producida desde múltiples lugares y experiencia de conquista del nuevo mundo, es fundamental en la gestación del imaginario euro-céntrico. En este sentido sostenemos que la interculturalidad en América Latina se origina simultáneamente en diferentes contextos y desde hace varios siglos, a través de los múltiples movimientos sociales, comerciales, culturales y de cooperación entre los diferentes pueblos y naciones en el mundo (Quijano, 1988; López y Küper, 2000; Tubino, 2002; Quilaqueo, Fernández y Quintriqueo, 2010).

En ese contexto, la interculturalidad surge tanto desde el ámbito social como institucional. En el ámbito social, surge primeramente desde el período colonial, de acuerdo al apartado anterior, como una estrategia evangelizadora de la Corona española y por detener la violencia y las consecuentes pérdidas, y de aumentar los súbditos del rey. Y luego, la interculturalidad aparece asociada a las reivindicaciones e insurgencias de movimientos sociales e indígenas en relación a las precarias condiciones de vida, desde el siglo XIX en adelante (Tubino, 2005a; Lepe-Carrión, 2012a, 2012b, 2012d).). En el ámbito institucional, la interculturalidad surge como una respuesta de los Estados-Nación a la educación escolar que demandan los pueblos indígenas, desde principios del siglo pasado (Ponce De León, Rengifo y San Martín, 2010; Quintriqueo y Muñoz, 2013). Así, la interculturalidad en América Latina surge desde los sectores sociales excluidos y más postergados del proyecto de modernización de los países, donde la mayoría de los indígenas son marginados, generando una fuerte inequidad social, tensiones, discriminación, racismo y desigualdades (Ferrão, 2010; Tubino, 2011). Dichos elementos son centrales en la historia de América Latina en la construcción de las repúblicas coloniales, donde la educación y la iglesia se constituyen en 
la base estratégica de la República para formar a los futuros ciudadanos fundados en la monoculturalidad, tanto para indígenas como para los no indígenas.

En este marco, la educación intercultural se sitúa en el contexto escolar, como una estrategia de los Estados-Nación para continuar el desarrollo social, educacional, político y económico, fundado en la lógica colonial euro-céntrica (Tubino, 2002; De Sousa, 2009). Desde este argumento, los movimientos sociales y políticos indígenas, específicamente las organizaciones y comunidades, reaccionan frente a la exclusión y el racismo, demandando mayor calidad y pertinencia a la educación escolar. Así, la educación intercultural, desde sus orígenes y hasta hoy día, continúa su rol funcionalista a los Estados-Nación, para colonizar a las nuevas generaciones desde el monoculturalismo euro-céntrico y el monolingüismo de las sociedades hegemónicas que conquistaron América Latina. En esta lógica, como hemos señalado, se plantea la educación intercultural como una educación dirigida especialmente a los indígenas, lo que se traduce en una educación de mala calidad. El otro problema es que la sociedad occidental que conforman las élites de América Latina, desde la conquista, pasando por los procesos de colonización y modernización, no se ha mostrado dispuesta a ver ni aceptar las diferencias sociales, culturales, lingüísticas y epistemológicas del conocimiento indígena en los procesos de formación escolar de la nueva ciudadanía. Este hecho, opera como un racismo epistemológico en las intenciones de dar sentido y contextualizar la educación intercultural para todos los ciudadanos, indígenas y no indígenas (Quintriqueo, Quilaqueo Y Torres, 2014), lo cual permanece en el tiempo de una forma institucionalizada y estructuralmente arraigada en la sociedad postmoderna (De Sousa, 2010).

Desde un marco general, una propuesta de educación intercultural debiera asumir los siguientes supuestos: 1) En relación con el proceso de enseñanza-aprendizaje se asume que las personas desarrollan habilidades y destrezas que le permiten vivir en una determinada comunidad, en la cual están inmersas; 2) Las destrezas y habilidades se relacionan con sistemas de significados que capacitan a las personas para comunicarse y con actividades prácticas que se orientan hacia la transformación del mundo natural, social y cultural; 3) Quienes participan de dichas situaciones lo hacen desde sus propias perspectivas y metas que son iguales, pero diferentes a las propuestas por las comunidades; 4) Las personas aprenden inmersas en un mundo social, aunque el acto de aprender se considere como un hecho predominantemente individual; 5) Las personas aprenden en la diferencia considerando estas como una riqueza de la producción humana, para mejorar la calidad de los aprendizajes, las oportunidades y la convivencia social; y 6) Las prácticas pedagógicas tienen por objeto la generación de una relación dialógica entre saberes populares, saberes indígenas y el conocimiento escolar (Quilaqueo y Quintriqueo, 2008).

De acuerdo a lo anterior, en el caso de Chile, la implementación de la educación intercultural requiere necesariamente del reconocimiento y legitimación de las minorías étnicas por parte del Estado, como pueblos con cultura propia y un patrimonio que debiera ser considerado en el currículum escolar (Quilaqueo, Quintriqueo y Cárdenas, 2005). En la actualidad, este proceso se está desarrollando con sistematicidad y consistencia, tanto en Chile como en el contexto latinoamericano, en donde se ha complejizado por la coexistencia de una gran diversidad de grupos étnicos, que aún mantienen vigentes sus culturas originarias; tales son los casos de Bolivia, Perú, Ecuador y México.

\section{Formación de profesores en educación intercultural}


La formación de profesores en educación intercultural, en contexto mapuche y especialmente en La Araucanía, se relaciona con las demandas y exigencias históricas, como una necesidad de las familias, comunidades y organizaciones sociales mapuches de lograr una educación escolar de calidad y pertinente a su realidad sociocultural; demandas identificadas con claridad desde principios del siglo XX (1920). Es así como la necesidad de formar profesores de origen indígena, que tengan por objeto desarrollar una educación escolar culturalmente pertinente, se concreta con los aportes de la Fundación Instituto Indígena, dependiente del Obispado de La Araucanía, 1980-1990. Dicha institución realiza estudios sobre la necesidad de innovación curricular de las instituciones encargadas de la formación de profesores, para adecuarlos a los requerimientos de la Ley Orgánica Constitucional de Educación, la que reconoce el nivel universitario sobre la formación de profesores en educación intercultural. Paralelamente considera las demandas sociales y educacionales de las organizaciones mapuches de fines del siglo pasado, las que remarcan la necesidad de formación universitaria de las nuevas generaciones, principalmente en el área de historia y la educación intercultural (Quintriqueo y Muñoz, 2013). Dichas demandas también son acogidas y formalizadas en la Comisión Especial de Pueblos Indígenas (CEPI), creada en 1989, la que tiene por objeto responder a las necesidades educacionales de las familias y comunidades indígenas en Chile.

Así, en Chile sólo a partir de 1992 la Universidad Católica de Temuco crea la carrera de Pedagogía en Educación Básica Intercultural (PEBI), cuyo objetivo es formar profesores capacitados para considerar las diferencias lingüistas y diversidad cultural de los estudiantes ( Desde el año 1992 al 2001, el ingreso de estudiantes a la carrera de Pedagogía en Educación Básica Intercultural se ha realizado a través de la Admisión Complementaria, dirigida principalmente a jóvenes mapuches: hombres y mujeres, donde el requisito fundamental es ser hablante del mapunzugun (lengua mapuche) y tener conocimientos sobre la cultura mapuche. Sin embargo, a partir del año 2000, además de la Admisión Complementaria, el ingreso es a través de la Admisión Regular, con lo cual, ingresan estudiantes de ascendencia mapuche y no mapuche). Este proceso se sustenta en el reconocimiento de la diferencia cultural y la coexistencia de los sistemas de saberes y conocimientos mapuches y occidental en el contexto escolar. En este marco, la formación de profesores tiene como propósito principal formar profesionales que puedan descubrir, reconocer y valorar las diferencias culturales para generar un diálogo entre sujetos pertenecientes a ambas culturas (Quilaqueo y Quintriqueo, 2008). La propuesta académica para la formación de profesores implica, necesariamente, el análisis de la complejidad que presenta la coexistencia de las racionalidades del conocimiento mapuche y no mapuche en el contexto escolar, como modos de construcción de sistemas de saberes para comprender y explicarse el mundo.

La finalidad de la formación de profesores en educación intercultural es conocer la cultura y sociedad mapuche para ser un actor social, generador de procesos educativos donde se asuma el patrimonio cultural propio de los estudiantes como contenido y finalidad educativa (Quintriqueo, 2013). El propósito es contribuir al desarrollo pleno de la identidad sociocultural en los estudiantes mapuches y no mapuches. En este proceso, comprender otras culturas y mejorar la calidad de la educación en contextos interculturales (La carrera actualmente se denomina 'Pedagogía en Educación Básica Intercultural en Contexto Mapuche', desde el 2002 en adelante. Esta se fortalece con la Ley Indígena № 19.253, de 1993, el Convenio de Colaboración MINEDUC-UCT iniciado en el año 1994 hasta hoy día. El propósito de dicho convenio es la formación de profesores de origen mapuche, capaces de implementar acciones educativas que permitan revertir el actual proceso de escolarización monocultural occidental imperante en contexto mapuche, a uno más pertinente y 
significativo a la realidad sociocultural de las familias y comunidades). El perfil de un profesor que egresa de Pedagogía en Educación Básica Intercultural en contexto mapuche se construye en relación a las siguientes áreas de formación establecidas por la Comisión Nacional de Acreditación de carreras de Pregrado (CNAP); (Esta es una carrera acreditada por la Comisión Nacional de Acreditación de Chile, hasta el año 2015):

i. El área de conocimiento científico y profesional, se relaciona con el conocimiento de las Ciencias de la Educación para el análisis e interpretación adecuada del medio social, natural y cultural. Así también se relaciona con el conocimiento sobre los fundamentos del trabajo pedagógico que permita tener una actitud crítica frente a las prácticas, el conocimiento educativo mapuche y el conocimiento disciplinario (escolar).

ii. El área de formación general responde al conocimiento de contenidos, métodos, materiales y recursos para la enseñanza, que posibilita generar climas de relación interpersonal en el aprendizaje de los estudiantes, formular metas de aprendizajes, implementar estrategias de evaluación para comprender el proceso de enseñanza y aprendizaje; generar autoevaluación del proceso pedagógico; y las competencias para el trabajo interdisciplinario.

iii. El área de formación de la especialidad, está vinculada con el conocimiento para generar propuestas curriculares para contextos interculturales; para diseñar, desarrollar y evaluar los distintos componentes de la enseñanza. En este sentido, la especialización se relaciona con asumir la revitalización de los valores y conocimientos socioculturales mapuches, esto igualmente ligado a conocimientos disciplinarios como contenidos de enseñanza del marco curricular.

iv. El área de formación actitudinal y valórica se relaciona con que el profesional demuestre actitudes positivas para atender la diversidad étnico-cultural; aplicar principios educativos interculturales; desarrollar un pensamiento crítico y reflexivo frente a las prácticas pedagógicas en escuelas situadas en contextos interculturales y asumiendo una visión cristiana del mundo y la educación como un proceso humanizador.

v. La formación práctica comprende una secuencia de actividades relacionadas con el aprendizaje de la docencia y la reflexión sobre la propia práctica, organizada en forma progresiva en el Plan curricular de la formación docente. Las experiencias prácticas incluyen actividades en terreno, cuenta con la participación de los principales responsables de las áreas de formación (de la disciplina y profesional) e involucra asociaciones con escuelas y profesores mentores en ellas. Culmina con una experiencia práctica intensiva de dos semestres. Se relacionan con competencias metodológicas y teóricas que el profesor debe dominar para comprender la cultura escolar, la realidad educativa, el currículum escolar, la socialización escolar y el trabajo pedagógico en contextos de relaciones interculturales.

El futuro profesor centra su interés en los saberes educativos mapuches y la aplicación del enfoque educativo intercultural, lo cual le permite democratizar la planificación, selección, enseñanza y aprendizaje de conocimientos educativos en contexto mapuche, con la implicación de todos los actores de los establecimientos escolares: familia, escuela y comunidad. Sin embargo, se observa que la formación descrita es limitada a una especialización para trabajar en contexto indígena, es funcionalista a las políticas de Estado en educación intercultural bilingüe, donde el concepto de diversidad étnica de la institución formadora se entiende casi exclusivamente para estudiantes de origen indígena. No obstante, la interculturalidad, desde la educación intercultural, debería orientarse a la 
formación para todos de los profesores como proyecto de vida que sobrepase, por una parte, la monoculturalidad de la escuela en tanto herencia colonial. Por otra parte, superar el concepto de diversidad cultural que en los hechos revela un eufemismo en su comprensión y explicación de la interculturalidad.

\section{Currículum escolar y educación intercultural}

El currículum escolar constituye un espacio, un campo de producción y creación de significados. La escuela es un centro donde se acoge todo tipo de acción educativa, consciente o no, contribuye a socializar y lograr la adquisición de contenidos en relación a una representación social del sujeto que enseña y del que aprende en el proceso educativo (Perrenoud, 2001). El currículum escolar en contexto mapuche está conformado por la selección de un conjunto de contenidos y finalidades educativas monoculturales decididos desde la institucionalidad del Estado, como conocimientos deseables de transmitir a las nuevas generaciones, a través de la educación escolar.

Sin embargo, un currículum para contextos indígenas debiera considerar un enfoque pedagógico intercultural, el cual no se conforma con la transmisión de contenidos, sino que busca una crítica epistemológica a los conocimientos educativos, las estrategias, metodologías y el paradigma euro-céntrico occidental que está a la base. Para AbdallahPretceille (1992), en la educación intercultural los conocimientos no pueden ser presentados como verdades inmutables y universales. La pedagogía intercultural exige una confrontación permanente de puntos de vista. Esto implica una capacidad de descentralización que lleve a una sistematización y estructuración de la educación para comunidades indígenas. Así, la reciprocidad de culturas y de las personas excluye la idea de un centro que representa el todo. La educación intercultural, por una parte, se entiende como una estrategia didáctica que tiene en cuenta ambas lógicas sociales y culturales en interrelación, paradigma sociocultural mapuche y occidental en el marco de sus respectivas epistemologías que están en la base del proceso de construcción del conocimiento educativo (Quintriqueo, 2013). Por otra parte, como enfoque intercultural en educación, permite el estudio y comprensión de la confrontación de personas pertenecientes a esferas culturales diferentes, lo que relativiza los conocimientos y obliga a construir un saber multipolarizado (Ouellet, 1991; Abdallah-Pretceille, 2001; Quintriqueo, 2010).

En efecto, el currículum intercultural tiene como finalidad la organización y sistematización de contenidos educativos propios de los estudiantes, transmitidos a partir de la socialización en el medio familiar y comunitario. Este proceso de explicitación de contenidos y finalidades educativas mapuches permitiría comprender y mejorar prácticas de escolarización en contextos interculturales (Quintriqueo, 2010). Además, revertir el problema de la generación de conflictos cognitivos, emocionales y de identidad étnica de los estudiantes de ascendencia mapuche como consecuencia de la escolarización monocultural. $\mathrm{Y}$, al mismo tiempo, instaurar un modelo dialógico intercultural en la definición de contenidos, finalidades educativas y procesos de escolarización contextualizados a la institucionalidad de las comunidades y de la escuela en contexto mapuche (Quintriqueo, 2010).

Desde una visión crítica del currículum, sustentada en los aportes de la Sociología de la Educación y de la Sociología del Conocimiento se considera vital los diversos campos y aspectos de la vida social, para sistematizar y comprender la educación y los contenidos educativos desde la práctica de significación. Campos y actividades tan diversas como la ciencia, la economía, la política, la salud, la alimentación, la educación y el currículo, son 
todos culturales en la medida en que las prácticas de significación constituyen una actividad fundamental de su existencia y funcionamiento (Magendzo, 1986; Tedesco, 2000; Perrenoud, 1994, 1998, 2001). En este sentido, los recientes aportes de las ciencias cognitivas y fundamentalmente en la perspectiva constructivista, pueden servir también de sustento consistente para una propuesta de educación intercultural. Por ejemplo cuando se señala: a) El conocimiento no es pasivamente recibido por el aprendiz, sino que activamente construido por éste, y b) La cognición tiene una función adaptativa y sirve para organizar el mundo experiencial y no tan solo para el descubrimiento de una realidad ontológica (Von Glaserfeld, 1991). Dentro de esta misma perspectiva se considera que el sujeto organiza las situaciones nuevas con estructuras de representación o de acciones precedentes, en las situaciones análogas y conservadas en la memoria desde que se construyen.

Así, desde una perspectiva curricular, las características socioculturales y lingüísticas que presentan las familias y comunidades mapuches, como son las nociones de tiempo, espacio, relación hombre-naturaleza, organización e identidad territorial, lengua, relación de parentesco y organización comunitaria, espiritualidad y significados simbólicos, son categorías de conocimientos centrales deseables de incorporar a una propuesta curricular intercultural (Quintriqueo y Maheux, 2004; Quintriqueo y Quilaqueo, 2006; Quintriqueo, 2010; Torres y Quilaqueo, 2010). Estos contenidos y finalidades educativas que emanan desde el conocimiento propio de las familias y comunidades mapuches, han logrado ser reconocidos y sistematizados en investigaciones del Centro de Investigación en Educación en Contexto Indígena e Intercultural (CIECII). Son saberes y conocimientos educativos que emergen de la memoria social mapuche y que pueden ser incorporados a las bases curriculares del Ministerio de Educación de Chile y los programas de formación en la Educación Superior.

\section{La interculturalidad: desafíos en educación}

El concepto de interculturalidad en la actualidad, habla no sólo del reconocimiento de la existencia de las culturas indígenas, sino que busca construir un diálogo entre éstas, basado en la calidad de la enseñanza y aprendizaje en relación con el saber indígena-occidental y viceversa. Es decir, un aprendizaje mutuo y la posibilidad de generar una universalidad, fruto de este diálogo intercultural (Fornet-Betancourt, 2001). Para ello es necesario comenzar problematizando el concepto de cultura y situarse críticamente frente al concepto clásico que la concibe como ente monolítico, unificado, inmutable y sin tensiones (Abdallah-Pretceille, 2001). Ello demanda tomar un concepto que explicite las contradicciones y tensiones al interior de cada universo cultural. En esta perspectiva, el sujeto perteneciente a una cultura posee la capacidad crítica de cuestionar no sólo la relación entre dos culturas, sino también la relación individual del sujeto con su propia cultura. Este proceso permite asumir que al interior de las distintas sociedades existen tensiones, conflictos y relaciones de poder susceptibles de ser modificados por la voluntad de los individuos y la sociedad en su conjunto. En efecto, es necesario asumir que cada una de sus culturas no son entes consolidados sino, más bien, que se configuran también como un proyecto a construir.

En efecto, entendemos la interculturalidad como un proyecto social, político, educacional y económico, basado en la necesidad de producir un diálogo de saberes, con el fin de poder incorporar los aportes de otras sociedades y culturas al desarrollo de los pueblos (Olivé, 2009; Quintriqueo, 2010; Quintriqueo, Quilaqueo y Torres, 2014). Dicho diálogo debe ser, necesariamente, contextualizado con las relaciones de dominación existentes entre 
indígenas y no indígenas en Chile, con el fin de desestructurar la matriz colonial de poder que hemos heredado de los encubrimientos socio-históricos del racismo. Se refiere a un diálogo que no cierra los ojos frente a las relaciones de poder existentes, sino por el contrario busca visibilizarlas, explicitarlas, hacerlas parte del diálogo desde una coconstrucción con los actores sociales (Chiodi y Bahamondes, 2002). La interculturalidad busca también hacerse cargo de las relaciones de opresión entre sociedades y no sólo constatarlas. El fin es lograr una superación de la trivialización de las identidades sociohistóricas de personas y grupos en América Latina. Este proceso permitiría constituir sistemas de relaciones más humanas, más comprensivas, capaces de reconocer las diferencias, ventajas y desventajas para configurar un paradigma alternativo: la interculturalidad crítica (Tubino, 2005a, 2005b; Viaña, 2010; Walsh, 2010). Así, desde este paradigma, la formación en educación básica intercultural y la educación intercultural, debe buscar que los estudiantes puedan ver en las diferencias no sólo una alternativa al modo de vida de la cultura de origen, sino también una alternativa posible de ser incorporada en la escuela (Essomba, 1999). Esto significa, la valorización de la identidad, los contenidos y finalidades educativas de los otros, para la formación de persona; construcción de conocimientos y competencias dialógicas entre el saber mapuche y occidental en el contexto escolar (Quilaqueo, Quintriqueo, Torres y Muñoz, 2014).

En síntesis, basados tanto en nuestras investigaciones como en la documentación normativa provenientes del MINEDUC (2005) y UNESCO (2006), ESSOMBA (2012), Quilaqueo, Quintriqueo, Torres Y Muñoz (2014), pensamos que las características de un programa de educación intercultural en contexto indígena y mapuche en lo específico, tiene como finalidad: 1) El diálogo entre sujetos que pertenecen a sociedades y culturas diferentes; 2) Contrarrestar la linealidad de la monoculturalidad; 3) Asumir el acción educativa mapuche (kimeltuwün), como base metodológica para concretar los principios de la educación intercultural, apoyada en los contenidos y finalidades educativas como una práctica de validación objetiva y subjetiva de lo propio, para superar el desprecio epistemológico del saber educativo mapuche en el contexto escolar; (4) Una acción educativa que favorece el descubrimiento de la diversidad cultural, la conciencia del valor positivo de la diversidad cultural y el respeto al patrimonio cultural local; (5) Una práctica pedagógica fundada en la conciencia crítica sobre la lucha contra el racismo y la discriminación; (6) Un conocimiento acerca del patrimonio cultural local, mediante la enseñanza de la historia, la geografía, la literatura, las lenguas, las artes, los temas científicos y tecnológicos; (7) Una comprensión y respeto a todos los pueblos, sus culturas, civilizaciones, valores y formas de vida, comprendidas las culturas indígenas nacionales como de otras naciones; (8) Un reconocimiento de la creciente interdependencia mundial de los pueblos y las naciones; (9) Una conciencia no sólo de los derechos sino también de los deberes mutuos que tienen los individuos, los grupos sociales y nacionales; (10) La colaboración activa y participativa de la familia y la comunidad en la planificación, desarrollo y evaluación de los procesos educativos; (11) Que el profesor debe conocer el contexto sociocultural de los estudiantes y estar dispuesto a aprender de sus experiencias educativas y socioculturales; y (12) Que el profesor debe estar consciente de los propios valores culturales que orientan la interpretación de las situaciones y problemas, así como la capacidad para reflexionar sobre la información y reevaluarla a la luz de diferentes perspectivas culturales y respetando diferentes patrones de pensamientos (Quilaqueo, Quintriqueo, Torres y Muñoz, 2014).

En conclusión, para el logro de estos objetivos de la formación del profesorado en educación intercultural, es necesario implementar un proceso continuo de acciones 
concretas, pero también de una constante reflexión sobre el mismo quehacer en las instituciones formadoras de profesores. En este marco, la formación del profesorado en educación intercultural en el contexto nacional necesita una formación sólida en investigación y un compromiso vocacional. La investigación debe permitir no solo identificar líneas de pensamiento que se sustenten en una crítica epistemológica, para superar la educación intercultural funcionalista, en contextos indígenas y la enseñanza monocultural encubierta en la escuela, en el medio familiar y social. Sino, también la indagación del desempeño docente tanto en la formación inicial como en el medio escolar.

\section{Bibliografía}

Abdallah-Pretceille, M. (1992). Vers une pédagogie interculturelle. Paris: Anthropos.

Abdallah-Pretceille, M. (2001). La educación intercultural. Barcelona: Idea Books.

Arias, J. \& Restrepo, E. (2010). "Historizando raza: propuestas conceptuales y metodológicas". Emancipación y crítica, 3, 45-64.

Baudelot, C. \& Leclerq, F. (2008). Efectos de la educación. Buenos Aires: Del estante editorial.

Bengoa, J. (1987). Historia del Pueblo Mapuche. Santiago de Chile: Ediciones Sur, Colección Estudios Históricos.

Boccara, G. (1996). "Notas acerca de los dispositivos de poder en la sociedad colonialfronteriza, la resistencia y la transculturación de los reche-mapuche del centro-sur de Chile (XVI-XVIII)". Revista de Indias, LVI (208), 659-95.

Boccara, G. (1999). "El poder creador: tipos de poder y estrategias de sujeción en la frontera sur de Chile en la época colonial”. Anuario de Estudios Americanos, LVI (1), 65-94.

Burns, K. (2007), "Desestabilizando la raza", En: Marisol De la Cadena (ed.), Formaciones de indianidad: articulaciones raciales, mestizaje y nación en América Latina. Popayán: Envión.

Cano, D. (2010). "La demanda educacional mapuche en el periodo reduccional (1883-1930). Revista Pensamiento Educativo", Volumen 46 y 47, (pp. 317-335). En Chiodi, F. \& Bahamondes, M. (2002). Una escuela: diferentes culturas. Santiago de Chile: LOM Ediciones.

Chiodi, F. \& Bahamondes, M. (2002). Una escuela: diferentes culturas. Santiago de Chile: LOM Ediciones.

De La Cadena, M. (2007). "Introducción", En Marisol De la Cadena (ed.), Formaciones de indianidad: articulaciones raciales, mestizaje y nación en América Latina. Popayán: Envión.

De sousa, B. (2009). Una epistemología del sur. La reivindicación del conocimiento y la emancipación social. México: CLACSO y Siglo XXI.

De sousa, B. (2010). Descolonizar el saber, reinventar el poder. Uruguay: Ediciones Trilce.

Essomba, M. (1999). Construir la escuela intercultural. Reflexiones y propuestas para trabajar la diversidad étnica cultural. Barcelona: Editorial GRAO. 
Essomba, M. (2012). Inmigración e interculturalidad en la ciudad. Principios, ámbitos y condiciones para una acción comunitaria intercultural en perspectiva europea. Barcelona: Graó.

Ferrão, M. (2010). Educación intercultural en América Latina: Distintas concepciones y tensiones actuales. Revista Estudios Pedagógicos XXXVI, N² 2: 333-342.

Fornet-Betancourt, R. (2001). Filosofía e interculturalidad en América Latina, Intento de introducción no filosófica pp. 63-73. En Heise M. (ed.) Interculturalidad; Creacción de un concepto y desarrollo de una actitud. Lima: Inversiones Hathuey S.A.C.

Freire, P. (2002). Pedagogía del Oprimido. Madrid: Ed: Siglo XXI.

Hanisch, W. (1974). Historia de la Compañía de Jesús en Chile. Buenos Aires: Editorial Francisco de Aguirre).

Jara, A. (1971). Guerra y sociedad en Chile: la transformación de la Guerra de Arauco y la esclavitud de los indios. Santiago: Editorial Universitaria.

Lepe-carrión, P. (2012a), "El Contrato Colonial de Chile. Crítica a la racionalidad impura, en la configuración racial del discurso científico, político y prácticas culturales en los siglos XVII, XVIII y XIX", Tesis inédita para optar al grado de Doctor en Filosofía, Pontificia Universidad Católica de Valparaíso: Instituto de Filosofía.

Lepe-carrión, P. (2012b). "Reflexiones en torno a la instalación de la colonialidad en Chile. Evangelización, educación y reproducción de la 'diferencia colonial' en el siglo XVII", En: José Solano y otros (ed.), Colonialidad/Decolonialidad del Poder/Saber. Miradas desde el Sur; Valdivia: Ediciones Universidad Austral de Chile, 223-42.

Lepe-carrión, P. (2012C), "Civilización y barbarie. La instauración de una 'diferencia colonial' durante los debates del siglo XVI, y su encubrimiento como 'diferencia cultural". Andamios, Revista de investigación social, 9 (20), 63-88.

Lepe-carrión, P. (2012d), "Biopolítica borbónica en Chile: el discurso antropológico sobre la ociosidad y el vagabundaje", En: Martín Díaz y Carlos Pescader (ed.), Descolonizar el presente: ensayos críticos desde el sur; General Rocca - Argentina: Publifadecs.

López, L., \& Küper, W. (2000). La educación intercultural bilingüe en América Latina: balance y perspectivas. Revista Iberoamericana de Educación, $\mathrm{N}^{\circ} 20$.

Magendzo, A. (1986). Currículum y Cultura en América Latina. Santiago, PIIE.

Merino, Me., Quilaqueo, D. \& Saiz, J. (2008). Una tipología del discurso de discriminación percibida en mapuches de Chile. Revista signos, 41(67), 279-297. Recuperado en 30 de abril de 2014, de http://www.scielo.cl/scielo.php?script=sci_arttext\&pid=S071809342008000200011\&lng=es\&tlng=es. 10.4067/S0718-09342008000200011.

Ministerio de Educación (2005): Orientaciones para la contextualización de Planes y Programas para la Educación Intercultural Bilingüe. Santiago de Chile.

Olivé, L. et al. (2009). Pluralismo epistemológico. La Paz: CLACSO.

Ouellet, F. (1991). L'éducation interculturelle. Essai sur le contenu de la formation des maîtres. Paris: Editions L'Harmattan.

Perrenoud, P. (1994). Métier d'élève et sens du travail scolaire. Paris: ESF édition.

Perrenoud, P. (1998). La construction des compétences à l'école. Paris: ESF édition. 
Perrenoud, P. (2001). "La formación de los docentes en el siglo XXI". Revista de Tecnología Educativa, 14(3), 503-523.

Poblete, R. (2009). Educación Intercultural en la Escuela de Hoy: reformas y desafíos para su Implementación. Revista latinoamericana de Educación Inclusiva, $\mathrm{n}^{\circ} 2$.

Ponce de León, M., Rengifo, F., \& San martín, E. (2010). “¿Demanda social por educación? Estadística, sociedad y alfabetización a partir del censo de la república de 1854". Pensamiento Educativo, Vols. 46-47, 2010. pp. 303-316.

Quijano, A. (1988). Modernidad, identidad y utopía en América Latina. Lima: Sociedad y Política.

Quilaqueo, D. \& Merino, M. (2003). "Estereotipos y prejuicio étnico hacia los mapuches en textos complementarios a la asignatura de Historia. Revista Campo Abierto, $\mathrm{N}^{\circ} 23$, pp.119-135.

Quilaqueo, D. \& Quintriqueo, S. (2008). "Formación docente en educación intercultural para contexto mapuche en Chile". Cuadernos interculturales, 6(10), 91-110.

Quilaqueo, D., Fernández, C. \& Quintriqueo, S. (2010). Interculturalidad en contexto mapuche. Argentina: Editorial Educo Universidad del Comahue.

Quilaqueo, D., Merino, M. \& Saiz, J. (2007). "Representación social mapuche e imaginario social no mapuche de la discriminación percibida”. Revista Atenea, № 496, pp.81-103.

Quilaqueo, D., Quintriqueo, S. \& Cárdenas, P. (2005). Educación, currículum e interculturalidad. Elementos sobre formación de profesores en contexto mapuche. Santiago de Chile: Facultad de Educación, Universidad Católica de Temuco, Frasis editores.

Quilaqueo, D., Quintriqueo, S., Torres, H. \& Muñoz, G. (2014). Saberes educativos mapuches: aportes epistémicos para un enfoque de educación intercultural. Revista Chungará (en prensa).

Quintriqueo S. \& Muñoz, G. (2013). Demandes éducationnelles socio-historique et interculturel dans le contexte mapuche. En Salas, R. et Le Bonniec F. «Les Mapuche à la Mode». Ed. L'Harmattan (en prensa).

Quintriqueo S. (2013). "Réflexions sur la formation des enseignants interculturelle dans le contexte de la communauté autochtone mapuche au Chili". En Gisèle Maheux et Roberto Gauthier (Ed). La formation des enseignants inuit et des Premières Nations. Problématiques et pistes d'action. Canadá: Presses de l'Université du Québeque.

Quintriqueo, S. \& Maheux, G. (2004), "Exploración del conocimiento sobre la relación de parentesco como contenido educativo para un currículum escolar intercultural en comunidades mapuche". Revista de Psicología, vol. XIII, 1, 73-91.

Quintriqueo, S. \& Mcginity, M. (2009), "Implicancias de un modelo curricular monocultural en la construcción de la identidad sociocultural de alumnos mapuches de la IX Región de la Araucanía, Chile". Estudios Pedagógicos,. 35, 173-188.

Quintriqueo, S. (2010). Implicancias de un modelo curricular monocultural en contexto mapuche. Santiago: Gráfica LOM.

Quintriqueo, S., \& Quilaqueo, D. (2006). "Conocimiento de relación de parentesco como contenido educativo para escuelas situadas en comunidades mapuches de Chile". Revista Cuadernos Interculturales, 7, 81-95. 
Quintriqueo, S., Quilaqueo, D., \& Torres, H. (2014) Contribución para la enseñanza de las ciencias naturales: saber mapuche y escolar. Educ. Pesqui., São Paulo (en prensa).

Tedesco, J. (2000). Educar en la sociedad del conocimiento. México: Fondo de Cultura Económica.

Torres, H. \& Quilaqueo, D. (2010). "Concepción de tiempo en el discurso educativo de los kimches”. En D. Quilaqueo, C. Fernández y S. Quintriqueo (Eds.), Interculturalidad en contexto mapuche (257-278). Neuquén, Chile: Educo.

Tubino, F. (2002). "Entre el multiculturalismo y la interculturalidad: más allá de la discriminación positiva" (pp.51-76). En Fuller, Norma (ed.): Intercultualidad y Política. Desafíos y posibilidades, Red para el Desarrollo de las Ciencias Sociales el Perú, Lima.

Tubino, F. (2005a). "La praxis de la interculturalidad en los Estados Nacionales Latinoamericanos". Cuadernos Interculturales, 3 (5), 83-96.

Tubino, F. (2011), "El nivel epistémico de los conflictos interculturales". Revista Construyendo Nuestra Interculturalidad, Vol. 6, Año 7, pp. 1-14.

Tubino, F.(2005b). "La interculturalidad crítica como proyecto ético-político', Encuentro continental de educadores agustinos" (enero 24-28, 2005). <https://oala.villanova.edu/congresos/educacion/lima-ponen-02.html >.

UNESCO (2006): Directrices de la UNESCO sobre la educación intercultural. Organización de las Naciones Unidas para la Educación, la Ciencia y la Cultura. Impreso en la UNESCO, París.

Viaña, J. (2010). "Fundamentos para una interculturalidad crítica", En: Luis Tapia y Catherine Walsh Jorge Viaña (ed.), Construyendo interculturalidad crítica; La Paz: Instituto Internacional de Integración del Convenio Andrés Bello, 97-133.Walsh, Catherine (2010), "Interculturalidad crítica y educación intercultural", En: Luis Tapia y Catherine Walsh Jorge Viaña (ed.), Construyendo interculturalidad crítica; La Paz: Instituto Internacional de Integración del Convenio Andrés Bello, 75-96.

Von Glasersfeld, E. (1991). "A Constructivistís View of Learning and Teaching”. In: R. Duit, F. GOLDBERG \& H. NIEDDERER (ed.) Research in physics learning: Theoretical issues and empirical studies. Proceedings of an international workshop. Kiel, Germany: IPN, 2939, 1991.

Walsh, C. (2010). "Interculturalidad crítica y educación intercultural”. In J. Viaña, L. Tapia \& C. Walsh (Eds.), Construyendo Interculturalidad Crítica (pp. 75-96). Bolivia: Instituto Internacional de Integración del Convenio Andrés Bello. 


\section{Autores}

Segundo Quintriqueo

Doctor en Educación, Profesor Asociado de la Universidad Católica de Temuco (UCT), Chile, Investigador Titular del CIECII, del Núcleo de Estudios Interétnicos e Interculturales (NEII) de la UCT e Investigador Asociado del Centre Interuniversitaire d’Études et de Recherches Autochtones (CIÉRA) de l'Université Laval, Québec, Canadá. Es autor principal de los artículos "Contribución para la enseñanza de las ciencias naturales: saber mapuche y escolar". Educ. Pesqui., São Paulo, Ahead of print, fev. 2014 y "Construcción de conocimiento mapuche y su relación con el conocimiento escolar". Estudios Pedagógicos, Vol.XXXIX, Nº 1: 199-216. E-mail: squintri@uct.cl

Daniel Quilaqueo

Doctor en Sociología, Profesor Titular de la Universidad Católica de Temuco. Investigador Responsable del Núcleo Milenio CIECII Investigador Titular del Núcleo de Estudios Interétnicos e Interculturales (NEII) de la UC Temuco. También es Investigador Asociado del Centre Interuniversitaire d'Études et de Recherches Autochtones (CIÉRA) de I'Université Laval, Québec, Canada. Sus últimas publicaciones: “Multiculturalidad e interculturalidad: desafíos epistemológicos de la escolarización desarrollada en contextos indígenas. Alpha [online]. 2013, n.37, pp. 285-300 y "Saberes educativos mapuches: aportes epistémicos para un enfoque de educación intercultural”. Revista Chungará (en prensa).E-mail: dquilaq@uct.cl

\section{Patricio Lepe-Carrión}

Doctor en Filosofía, Profesor de la Universidad Católica de Temuco. Investigador del CIECII e Investigador Adjunto del Instituto de Estudios Sociales y Culturales PENSAR (PUJColombia). Autor de artículos científicos, capítulos de libros, y columnas en revistas electrónicas relacionadas con Estudios Poscoloniales, Estudios Culturales, Programa de Investigación Modernidad/Colonialidad en América Latina, Raza, Racismo y Etnicidad, Historia y Crítica del período colonial e independiente de Chile, Interculturalidad y Biopolítica. E-mail: plepe@uct.cl

Enrique Riquelme

Doctor en Educación, Profesor de la Universidad Católica de Temuco. Docente de la Facultad de Educación e Investigador joven del CIECII. Entre las publicaciones recientes se encuentran: "Análisis Factorial Confirmatorio de la Escala de Habilidades Sociales de Gismero". Revista Latinoamericana de Psicología. "Improving Emotional Competence Through Mediated Reading: Short Term Effects of a Children's Literature Program". Mind, Culture, and Activity, 20(3),226-239. E-mail: eriquelme@uct.cl 
Maritza Gutiérrez

Doctora en Ciencias, Profesora Asistente de la Universidad Católica de Temuco. Profesora asistente de la Universidad Católica de Temuco e investigadora asociada del CIECII. Investigadora responsable del proyecto DGIUCT "Los tintes vegetales mapuches como colorantes vitales con sentido y significado para la enseñanza de la biología en contexto rural mapuche y no mapuche", dentro de sus publicaciones: "Conocimientos sobre colorantes vegetales: contenidos para la educación intercultural en ciencias". Revista Perfiles Educativos. E-mail: mcgutierrez@uct.cl

Fernando Peña-Cortés

Doctor en Ciencias Ambientales, Profesor Titular de la Universidad Católica de Temuco. Actualmente se desempeña como Coordinador del Laboratorio de Planificación Territorial (ww.lpt.cl) y Director del Programa de Magister en Planificación y Gestión Territorial de la Universidad Católica de Temuco. Sus principales trabajos se basan en el ámbito de Análisis y Planificación Territorial. Ha desarrollado importantes proyectos FONDECYT, FONDEF, INNOVA fpena@uctemuco.cl

\section{Agradecimientos}

Agradecemos al Fondo Nacional de Desarrollo Científico y Tecnológico FONDECYT por el financiamiento de los proyectos de investigación $\mathrm{N}^{\circ} 1140490$ "Conocimientos geográficos y territoriales mapuches: una base para la formulación de contenidos educativos interculturales pertinentes y contextualizados", N 1140562 "Construcción social del conocimiento educativo mapuche: doble racionalidad y desafíos para una escolarización intercultural" y N 1140864 "Desarrollo de Competencia Comunicativa Intercultural (CCI) en estudiantes universitarios de las regiones del Maule, Bio-Bio y La Araucanía". 